\title{
The EMBnet International Conference 2020: Bioinformatics Approaches to Precision Research
}

\author{
Domenica D’Elia1 ${ }^{1 凶}$, Cesar Bonavides-Martinez², Erik Bongcam-Rudloff ${ }^{3}$, Emiliano Barreto-Hernandez ${ }^{4}$, \\ Aspasia Efthimiadou ${ }^{5}$, Eleni Papakonstantinou ${ }^{6}$, Dimitrios Vlachakis ${ }^{6,7,8}$, Lubos Klucar ${ }^{9}$ \\ 'Institute for Biomedical Technologies, National Research Council, Bari, Italy \\ ${ }^{2}$ Universidad Nacional Autónoma de México (UNAM), Mexico City, Mexico \\ ${ }^{3}$ Swedish University of Agricultural Sciences, Uppsala, Sweden \\ ${ }^{4}$ Bioinformatics Center, Biotechnology Institute, Universidad Nacional de Colombia, Bogota, Colombia \\ ${ }^{5}$ Hellenic Agricultural Organization-Demeter, Institute of Soil and Water Resources, Department of Soil Science of Athens, Lycovrisi, \\ Greece \\ ${ }^{6}$ Laboratory of Genetics, Department of Biotechnology, School of Applied Biology and Biotechnology, Agricultural University of \\ Athens, Athens, Greece \\ ${ }^{7}$ University Research Institute of Maternal and Child Health \& Precision Medicine, and UNESCO Chair on Adolescent Health Care, \\ National and Kapodistrian University of Athens, "Aghia Sophia" Children's Hospital, Athens, Greece \\ ${ }^{8}$ Division of Endocrinology and Metabolism, Center of Clinical, Experimental Surgery and Translational Research, Biomedical \\ Research Foundation of the Academy of Athens, Athens, Greece \\ ${ }^{9}$ Institute of Molecular Biology, SAS, Bratislava, Slovakia \\ Competing interests: DD none; CBM none; EBR none; EBH none; AE none; EP none; DV none; LK none
}

\section{Abstract}

In this paper, we first summarise the 2020 International Conference of EMBnet - The Global Bioinformatics Network on "Bioinformatics Approaches to Precision Research", held on 23th-24th September 2020, and then briefly introduce the main topics and contributions presented at the Conference and published in this supplement issue. The Conference's main aim was to share the knowledge and scientific achievements of EMBnet members to prompt the cross-fertilisation of ideas and collaborations. The title reflects the type of contributions presented (20 in total), covering a wide range of cutting-edge research topics for which advanced bioinformatics methods and precision approaches are essential, and training in Bioinformatics. As for many other organisations, 2020 was the first year that the EMBnet Annual General Meeting was not in presence but ran on a virtual meeting platform because of the pandemic's travel restrictions to moves. If this situation represented a limitation to our social activities, it allowed us to offer the possibility to attend the Conference to a larger number of researchers (i.e., 190 from 38 different countries). Among all registered people, 148 were following the conference presentations on both days. Under the authors' permission, presentations were recorded and are available at the EMBnet Conference 2020 playlist on YouTube, whereas the programme is available at the EMBnet Conference 2020 Google Drive folder.

\section{EMBnet Conferences in a nutshell}

EMBnet - The Global Bioinformatics Network ${ }^{1}$ is the first and oldest network established in Europe by researchers in Bioinformatics. It was constituted in 1988 as the "European Molecular Biology Network" by a handful of European countries to distribute and provide European research institutes with access to sequence data and bioinformatics tools, to connect molecular biologists and bioinformaticians within Europe and to provide education and training in Bioinformatics.
In 2008 EMBnet celebrated its 20th anniversary with an international conference on "Leading Applications and Technologies in Bioinformatics". This Conference was the first meeting organised by the network that was open to the international scientific community outside EMBnet (D'Elia et al., 2009).

Since then, each year, the Annual General Meeting (AGM) of the Network has always been accompanied by an open conference, a workshop or a school on cutting-edge challenges in the bioinformatics field and usually organised in collaboration with other network organisations or societies. With the Iberoamerican

${ }^{1}$ https://www.embnet.org/wp/about/

(c) 2021 D'Elia et al.; the authors have retained copyright and granted the Journal right of first publication; the work has been simultaneously released under a Creative Commons Attribution Licence, which allows others to share the work, while acknowledging the original authorship and initial publication in this Journal. The full licence notice is available at http://journal.embnet.org. 
Society for Bioinformatics (SoIBio) ${ }^{2}$, EMBnet organised a big conference in Cancun, Mexico, in 2009 (LopezBojorquez et al., 2010) and in 2018 in Viña del Mar (Chile), celebrating the 30th anniversary of EMBnet, also with the collaboration of the International Society for Computational Biology (ISCB) $)^{3}$. Other partners were the project EMBRACE (Gisel and BongcamRudloff, 2010), STATEGRA ${ }^{4}$, AllBio ${ }^{5}$, the COST Action SeqAhed (Attwood et al., 2011), the COST Action CHARME (CA15110) and NETTAB ${ }^{6}$, the Network Tools and Applications in Biology workshops series in Bioinformatics. In 2019 the EMBnet AGM was organised in the Republic of North Macedonia in conjunction with the ICT Innovation Conference 20197. On this occasion, EMBnet organised as a satellite event of the main Conference a workshop on "Big Data Analysis and Reproducibility: new challenges and needs of the postgenomic era" (Efthimiadou et al., 2019).

Plans for the EMBnet AGM 2020 were in preparation when the SARS-COV-2 invaded Europe and immediately after the American and African continents as a tsunami. There were no clues on how the pandemics could have been evolving in the upcoming months. The decision was taken in July 2020 to involve as much as possible the EMBnet members in an open and virtual scientific event where each member could present new ideas and projects, research activities and achievements. The main aim was to offer the members the possibility to share their work inside and outside the EMBnet community to prompt the cross-fertilisation of ideas and collaborations. The Conference attracted many researchers from all over the world, most of them being young researchers and students who actively participated in the scientific discussion of the research presented. The enthusiasm was contagious, and the atmosphere the one of a community that share common interest and passion for its work with optimism and willingness to do although the COVID-19 pandemic. As organisers of the EMBnet Conference 2020, we thank the speakers and attendants for their contribution to its success.

\section{EMBnet Conference 2020: numbers}

The Conference was an entirely digital event, and for this reason, we did not need to ask for a registration fee. Although the short anticipation of the conference programme, the number of registration was 190. Among all registered people, 148 followed the conference presentations on both days from 38 different countries inside and outside of Europe, such as Algeria, Brazil, Canada, Chile, China, Colombia, Costa Rica, Ecuador, India, Malaysia, Mexico, Nepal, Nigeria, Pakistan,
Russia, Saudi Arabia, Serbia, Sri Lanka, Taiwan, United Kingdom, United States of America.

The Conference daily sessions were organised to start at $3 \mathrm{pm}$ and end at $8 \mathrm{pm}$ (CEST) and using the Zoom Platform ${ }^{8}$. The time slot was fixed taking into consideration the time zone on diverse continents. Nevertheless, we recorded all presentations and made them available in the form of a playlist on YouTube ${ }^{9}$ to allow access at any time for people who could not follow live the Conference. These video presentations are included also in the digital version of this EMBnet. journal issue.

The programme and presentations files were deposited in a Google Drive folder dedicated to the Conference and open to participants under presenters' permission. A Slack Channel was also created and made available to registered people for timely communications with the programme committee members and speakers before, during and after the Conference.

The authors submitted contributions to the Programme Committee evaluation as short abstracts. Selected Works, in total 20, are published in this EMBnet Journal Supplement as Proceedings of the Conference.

\section{The EMBnet Conference 2020 sci- entific programme}

The Conference was open by Domenica D'Elia, the Chair of the EMBnet Executive Board, who welcomed participants and briefly presented EMBnet, its activities, mission and products ${ }^{10}$.

The first session was introduced and chaired by Professor Erik Bongcam-Rudloff, Chair of the EMBnet Project Committee on Education \& Training in Bioinformatics. Presentations of the first conference session gave a multifaceted overview of how bioinformatics precision approaches can be applied to various domains for sustainable development, in agriculture and animal farm, and to human health for more effective therapeutic strategies.

The session was opened by a presentation of Professor Dimitrios Vlachakis, member of the EMBnet Executive Board, on a holistic approach of systems biology and bioinformatic analysis on breast milk exosome. He demonstrated how breast milk molecular fingerprinting could pave the way to shed light on the underlying genetics and epigenetics that a mother can transmit to her children.

Dr Tomas Klingström presented the development of the Gigacow infrastructure ${ }^{11}$ for precision dairy farming and the research opportunities presented to researchers at SLU and international collaborators.

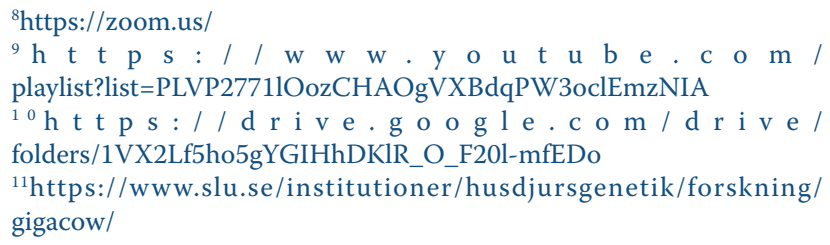

\author{
${ }^{2} \mathrm{http}$ //www.soibio.org/ \\ ${ }^{3}$ https://www.iscb.org/iscb-
${ }^{4}$ http://www.stategra.eu/ \\ ${ }^{5}$ http://www.allbioinformatics.eu/d
${ }^{6}$ http://www.igst.it/nettab/2016/ \\ ${ }^{7}$ http://www.ictinnovations.org/11th-international-conference-ict- \\ innovations-2019
}




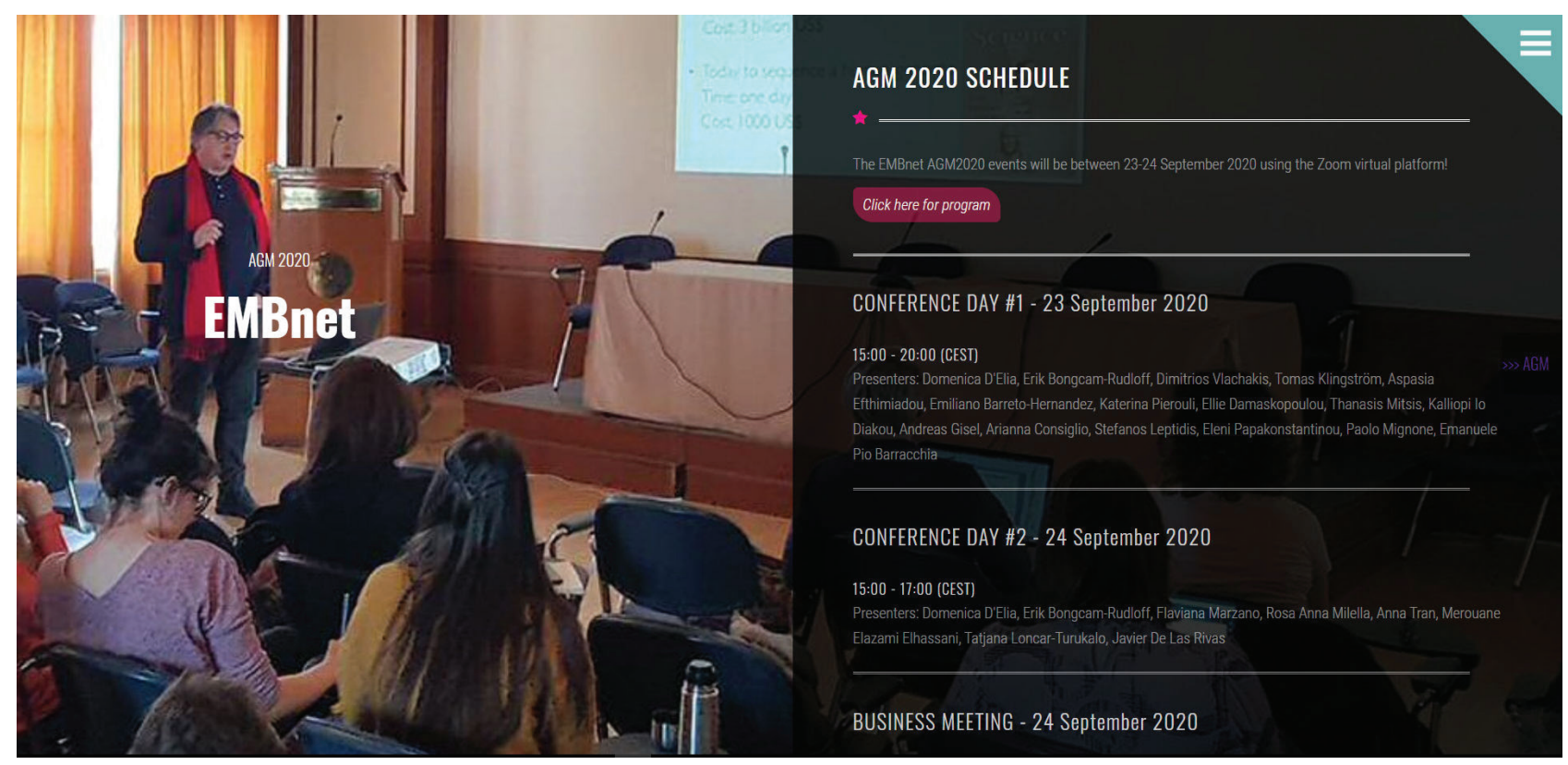

Figure 1. The EMBnet Conference 2020 web site.

Dr Aspasia Efthimiadou, Chair of the Publicity \& Public Relations Project Committee of EMBnet, exposed a case study on cotton cultivation to demonstrate how Neural Network-based Decision Support Systems, combined with data analysis, can be considered the future of sustainable agriculture.

Professor Emiliano Barreto-Hernandez, Treasurer and member of the EMBnet Executive Board, presented SGIG, the Genomic Information Management System, developed to integrate clinical, epidemiological, laboratory, and genomic data for precision epidemiology of multi-drug resistance bacteria in Colombia.

Finally, Dr Stefanos Leptidis, presented a methodology for identifying cell types using their single-cell micro RNA (miRNA) profile coupled to their predicted targets from various miRNA target prediction algorithms. This method is helpful to elucidate the intricate cellular interactions and regulatory pathways involved in organ-specific pathologies for the development of precision therapeutic approaches.

The second session on day one was chaired by Prof. Dimitrios Vlachakis. Themes treated spanned from genome regulation by long non-coding RNAs (ncRNAs) presented by Katerina Pierouli, to how the combination of Genome-Wide Association Studies (GWAS), Single Nucleotide Polymorphisms (SNPs), and methylation profiles analysis will provide the possibility to investigate mechanisms of epigenetic inheritance in children following exposure to abuse (presenter Ellie Damaskopoulou). On another side, Thanasis Mitsis illustrated how GWAS could be used to get insights into the complex interplay of nuclear receptor transcriptional networks to elucidate their contribution to the maintenance of cell homeostasis. Andreas Gisel, a member of the Education \& Training Project Committee of EMBnet, talked about the 3'TagSeq technology for transcriptomics studies, underlining how this technology gives not only precise information about gene expression but also for alternative annotation in the 3'UTR of genes. Arianna Consiglio, illustrated a study on the expression profile of non-coding RNAs in coronaviruses that demonstrates a possible action of RNA interfering on the human immune system response. Eleni Papakonstantinou, exposed a computational drug design strategy developed to discover the most potent molecules with an inhibitory effect on the helicase function and the viral replication cycle of the Yellow Fever Virus.

The third session, chaired by Prof. Emiliano BarretoHernandez, included two presentations illustrating applications of machine learning techniques to Big Data analysis for i) gene regulatory network reconstruction, presented by Paolo Mignone and using transfer learning techniques; and for ii) the prediction of relationships between ncRNAs and human diseases, presented by Emanuele Pio Barracchia, exploiting multi-type hierarchical clustering techniques.

The second day of the Conference was chaired by Lubos Klucar, Secretary of the EMBnet Executive Board, and included scientific presentations and a space dedicated to education and training.

The first presentation was by Flaviana Marzano, exposing a combined approach (in silico and lab experimental validation) demonstrating that plant micro RNAs can interfere with human IncRNAs to control cancer genes expression. On another side, Rosa Anna Milella exposed the results of a nutrigenomics study underlining the effects of grape intake on human gene expression, cell signalling pathways, and many ncRNAs. Anna Tran talked about in silico characterisation of the gene repertoires of immunoglobulins (IGs) and $\mathrm{T}$ cell receptors (TRs) of various inbred laboratory strains of M. musculus to design and develop or adapt highperformance software tools and a methodology to carry 
out the annotation of the loci IG and TR of the mouse strains with a "Gold standard" quality (equivalent to the manual annotation). Merouane Elazami Elhassani presented an approach based on deep neural networkbased models trained in a supervised manner, which automatically learns features from annotated IG and TR genes to predict the L-PART1 exon (the first exon of IG and TR variable $\mathrm{V}$-GENE).

\section{Education \& Training Bioinformatics session}

Invited speakers were Tatjana Loncar-Turukalo, Coordinator of the Short-Term Scientific Mission (STSM) programme of the COST Action CA18131 ML4Microbiome $^{12}$ (Statistical and machine learning techniques in human microbiome studies); Dr Javier De Las Rivas, President of SoIBio, the Iberoamerican Society for Bioinformatics; and Prof. David Coornaert of the University College "Haute Ecole en Hainaut Campus Technique”, in Mons (BE).

Dr Tatjana Loncar-Turukalo exposed the aims of the COST Action ML4Microbiome ${ }^{13}$ with a focus on training and education activities and possible opportunities for participation and collaboration. Dr Javier De Las Rivas presented "Advancement of Bioinformatics and Computational Biology in Latin America: SoIBio and other scientific networks" (De Las Rivas et al., 2019), making an overview of the history and current state of research in Bioinformatics in Latin America and underlining the role of SoIBio as a leading forum to join efforts of many scientists from LA to accelerate the datadriven biology research in LA and also a sustainable capacity-building programme based on education and training in Bioinformatics in collaboration with the CABANA project ${ }^{14}$. Prof. David Coornaert was invited along with two students of the University College Haute Ecole en Hainaut in Mons to present the programme of collaboration with EMBnet to train bachelor students inside the ERASMUS programme. His presentation was concluded by a nice presentation of the two students, Cyril Radermecker and Ahmed Kanfoud, about their ERASMUS stage in 2020 across the EMBnet Node in Greece under the supervision of Prof. Dimitrios Vlachakis.

The Conference ended at $5 \mathrm{pm}$. The EMBnet Executive Board thanked the speakers and present people for their contributions and participation with the promise to keep in touch for upcoming events or potential collaborations.

After the Conference, EMBnet members continued with the programme of the Annual General Meeting, including the Executive Board and Project Committees

\footnotetext{
${ }^{12}$ https://www.cost.eu/actions/CA18131/\#tabs|Name:overview

${ }^{13}$ https://www.ml4microbiome.eu/

${ }^{14}$ https://www.cabana.online/
}

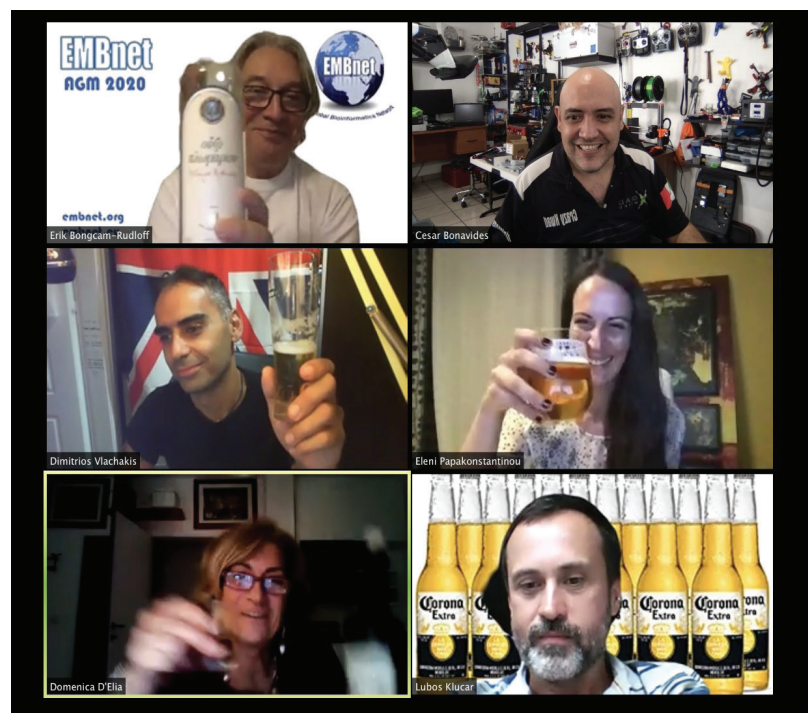

Figure 2. The survivors toasting at the success of the EMBnet Conference and of the AGM 2020.

activity reports, the presentation of the latest achievements and plans for the EMBnet Journal and the discussion on priorities and plans for 2020/2021. A virtual toast among some of the Operational Board's members (Fig. 2), survived at the intense last day activities, the success of the Conference and the pleasure to stay all together again, although geographically separate, with the wish that in 2021, EMBnet members can meet again in person to exchange true hugs that have been missed so much to all us.

\section{References}

1. Attwood T, Bongcam-Rudloff E, Gisel A (2011) SEQAHEAD COST Action BM1006: Next Generation Sequencing Data Analysis Network. EMBnet.journal, 17.A:7-9. http://dx.doi. org/10.14806/ej.17.1.218

2. D’Elia D, Gisel A, Eriksson NE, Kossida S, Mattila K et al. (2009) The 20th anniversary of EMBnet: 20 years of bioinformatics for the Life Sciences community. BMC Bioinformatics 10, Suppl 6:S1 doi: http://dx.doi.org/10.1186/1471-2105-10-S6-S1

3. De Las Rivas J, Bonavides-Martínez C, Campos-Laborie FJ (2019) Bioinformatics in Latin America and SolBio impact, a tale of spin-off and expansion around genomes and protein structures. Briefings in Bioinformatics, 20(2):390-397. http://! dx.doi.org/10.1093/bib/bbx064

4. Efthimiadou A, Papakonstantinou E, Vlachakis D, D'Elia D, Bongcam-Rudloff E (2019) Big Data analysis and reproducibility: new challenges and needs of the post-genomics era. In Springer Communications in Computer and Information Science book series. ICT Innovations 2019 Web proceedings: 253-257. ISSN 1857-7288

5. Gisel A, Bongcam-Rudloff E (2010) EMBRACE workshop "NEXT GENERATION SEQUENCING II". EMBnet.journal, 16.A:5-7. http://dx.doi.org/10.14806/ej.16.1.176

6. Lopez-Bojorquez Lucia et al. (2010) Report on the International Conference \& Meetings EMBnet-RIBio 2009. EMBnet.journal, 16.A:3-5. http://dx.doi.org/10.14806/ej.16.A.155 